DESY 97 - 221

ISSN $0418-9833$

November 1997

hep-th/9711119

\title{
ALTERNATIVE SOLUTION OF THE PATH INTEGRAL FOR THE RADIAL COULOMB PROBLEM
}

\author{
Christian Grosche \\ II. Institut für Theoretische Physik \\ Universität Hamburg, Luruper Chaussee 149 \\ 22761 Hamburg, Germany
}

\begin{abstract}
In this Letter I present an alternative solution of the path integral for the radial Coulomb problem which is based on a two-dimensional singular version of the Levi-Civita transformation.
\end{abstract}


Ever since the development of the path integral by Feynman [1] it has been challenge to calculate the propagator for the Coulomb potential. Due to Feynman himself were the evaluation of the path integral for the free particle and the harmonic oscillator, both belonging to a larger class of path integrals which are called Gaussian Path Integrals because they are based on the general quadratic Lagrangian. Nowadays more path integrals which go beyond the Gaussian form are known. They are the path integral solutions related to the radial harmonic oscillator [2], also called Besselian Path Integral, and the path integral solutions related to the Pöschl-Teller and modified Pöschl-Teller potential [3], also called Legendrian Path Integrals. These Basic Path Integrals are the building blocks for a comprehensive Table of Path Integrals [4, 5].

Since the path integral solutions of the one-dimensional and radial harmonic oscillator were known for a long time, it was a challenge to evaluate the path integral for the Coulomb potential, one of the most important physical system in quantum mechanics. In 1979 Duru and Kleinert [6, 7] succeeded in its calculation by means of a transformation known from astronomy [8], and now called space-time transformation, "Duru-Kleinert" transformation, time substitution, etc. [9]-22]. 1]

In this contribution I would like to present an alternative evaluation of the radial path integral of the Coulomb problem which is based on a singular version of the Levi-Civita transformation, which can be seen as a two-dimensional version of the Kustaanheimo-Stiefel transformation [8], as it has been used for the two-dimensional Coulomb problem [7, 20]. Let us consider the path integral for the Coulomb problem (C) in radial coordinates in three dimensions, where the propagator in $\mathbb{R}^{3}$ has been separated according to

$$
K^{C}\left(\mathbf{x}^{\prime \prime}, \mathbf{x}^{\prime} ; T\right)=\sum_{l \in \mathbb{N}} \sum_{n=-l}^{l} K_{l}^{C}\left(r^{\prime \prime}, r^{\prime} ; T\right) Y_{l}^{n *}\left(\vartheta^{\prime}, \varphi^{\prime}\right) Y_{l}^{n *}\left(\vartheta^{\prime \prime}, \varphi^{\prime \prime}\right)
$$

and $l$ is the usual angular momentum number, therefore

$$
K_{l}^{C}\left(r^{\prime \prime}, r^{\prime} ; T\right)=\frac{1}{r^{\prime} r^{\prime \prime}} \int_{r\left(t^{\prime}\right)=r^{\prime}}^{r\left(t^{\prime \prime}\right)=r^{\prime \prime}} \mathcal{D} r(t) \exp \left\{\frac{\mathrm{i}}{\hbar} \int_{t^{\prime}}^{t^{\prime \prime}}\left[\frac{m}{2} \dot{r}^{2}+\frac{e^{2}}{r}-\hbar^{2} \frac{l(l+1)}{2 m r^{2}}\right] d t\right\} \text {. }
$$

Similarly as in [6] I insert a factor "one" $(\epsilon=T / N)$ :

$$
\begin{aligned}
1= & \sqrt{\frac{m}{2 \pi \mathrm{i} \hbar T}} \int_{\mathbb{R}} d y^{\prime \prime} \exp \left(-\frac{m\left(y^{\prime \prime}-y^{\prime}\right)^{2}}{2 \mathrm{i} \hbar T}\right) \\
= & \int_{\mathbb{R}} d y^{\prime \prime} \lim _{N \rightarrow \infty}\left(\frac{m}{2 \pi \mathrm{i} \hbar \epsilon}\right)^{N / 2} \prod_{j=1}^{N-1} \int_{\mathbb{R}} d y_{j} \exp \left(\frac{\mathrm{i} m}{2 \epsilon \hbar} \sum_{j=1}^{N}\left(y_{j}-y_{j-1}\right)^{2}\right) .
\end{aligned}
$$

\footnotetext{
${ }^{1}$ Actually, the approach of Duru and Kleinert goes back to an idea by A.Barut presented in an informal seminal talk in Trieste 1978.

The Kustaanheimo-Stiefel transformation is in fact a special case of the Hurwitz transformation which works in the dimensions $1,2,4,8$.
} 
We obtain for the path integral (2) in the usual way

$$
\begin{aligned}
K_{l}^{C}\left(r^{\prime \prime}, r^{\prime} ; T\right) & =\frac{1}{r^{\prime} r^{\prime \prime}} \int_{\mathbb{R}} d y^{\prime \prime} \int_{r\left(t^{\prime}\right)=r^{\prime}}^{r\left(t^{\prime \prime}\right)=r^{\prime \prime}} \mathcal{D} r(t) \int_{y\left(t^{\prime}\right)=y^{\prime}}^{y\left(t^{\prime \prime}\right)=y^{\prime \prime}} \mathcal{D} y(t) \\
& \times \exp \left\{\frac{\mathrm{i}}{\hbar} \int_{t^{\prime}}^{t^{\prime \prime}}\left[\frac{m}{2}\left(\dot{r}^{2}+\dot{y}^{2}\right)+\frac{e^{2}}{r}-\hbar^{2} \frac{l(l+1)}{2 m r^{2}}\right] d t\right\} .
\end{aligned}
$$

I now consider in $\mathbb{R}^{2}$ the mapping

$$
A(\mathbf{u})=\left(\begin{array}{cc}
u_{1} & u_{2} \\
-u_{2} & u_{1}
\end{array}\right), \quad \mathbf{u}=\left(\begin{array}{l}
u_{1} \\
u_{2}
\end{array}\right) \in \mathbb{R}^{2} .
$$

$A(\mathbf{u})$ has the properties

$$
A(\mathbf{u}) \cdot \mathbf{u}=\left(\begin{array}{c}
u_{1}^{2}+u_{2}^{2} \\
0
\end{array}\right), \quad A^{t}(\mathbf{u}) \cdot A(\mathbf{u})=\left(u_{1}^{2}+u_{2}^{2}\right) \mathbb{1}=|\mathbf{u}|^{2} \mathbb{1} .
$$

Therefore $A: \mathbb{R}^{2} \mapsto \mathbb{R}^{+}$. Hence $A$ is not one-to-one, and it is not allowed to use the transformation $A$ in the path integral (4) in a naïve way. Instead, following [20], we must define it on midpoint coordinates in the following way $(i=1,2 ; j=1, \ldots, N)$ :

$$
\begin{aligned}
& \Delta r_{j}=2\left(\bar{u}_{1, j} \Delta u_{1, j}+\bar{u}_{2, j} \Delta u_{2, j}\right), \quad \bar{u}_{i, j}=\frac{1}{2}\left(u_{i, j}+u_{i, j-1}\right), \\
& \left.\Delta \mathbf{u}_{j}=2\left(-\bar{u}_{2, j} \Delta u_{1, j}+\bar{u}_{1, j} \Delta u_{2, j}\right), \quad \Delta u_{i, j}=u_{i, j}-u_{i, j-1}\right) \text {. }
\end{aligned}
$$

We then have $\left(\Delta r_{j}\right)^{2}+\left(\Delta y_{j}\right)^{2}=4\left(\overline{\mathbf{u}}_{j}\right)^{2}\left(\Delta \mathbf{u}_{j}\right)^{2}$ and $A^{t}\left(\overline{\mathbf{u}}_{j}\right) \cdot A\left(\overline{\mathbf{u}}_{j}\right)=\left(\overline{\mathbf{u}}_{j}\right)^{2} \mathbb{1}$. The Jocobian $J(\mathbf{u})$ of the transformation $A^{t}\left(\overline{\mathbf{u}}_{j}\right)$ for all $j=1, \ldots, N$ reads $J\left(\overline{\mathbf{u}}_{j}\right)=4\left(\overline{\mathbf{u}}_{j}\right)^{2}$, and we define $\bar{r}_{j}=\left(\overline{\mathbf{u}}_{j}\right)^{2}$ for all $j$. Taking into account this midpoint definition in the path integral (4) we obtain by performing the transformation $r \mapsto \mathbf{u}$ be means of $A\left(\overline{\mathbf{u}}_{j}\right)$

$$
\begin{aligned}
& K_{l}^{C}\left(r^{\prime \prime}, r^{\prime} ; T\right)=\frac{1}{4 r^{\prime} r^{\prime \prime}} \int_{\mathbb{R}} d y^{\prime \prime} \lim _{N \rightarrow \infty}\left(\frac{2 m}{\pi \mathrm{i} \hbar \epsilon}\right)^{N} \int_{\mathbb{R}^{2}}\left(\overline{\mathbf{u}}_{1}\right)^{2} d \mathbf{u}_{1} \ldots \int_{\mathbb{R}^{2}}\left(\overline{\mathbf{u}}_{N-1}\right)^{2} d \mathbf{u}_{N-1} \\
& \quad \times \exp \left[\frac{\mathrm{i}}{\hbar} \sum_{j=1}^{N}\left(\frac{2 m}{\epsilon}\left(\overline{\mathbf{u}}_{j}\right)^{2}\left(\Delta \mathbf{u}_{j}\right)^{2}-\frac{\epsilon \hbar^{2} l(l+1)}{2 m\left(\overline{\mathbf{u}}_{j}\right)^{2}\left(\overline{\mathbf{u}}_{j-1}\right)^{2}}+\frac{\epsilon e^{2}}{\left(\overline{\mathbf{u}}_{j}\right)^{2}}-\Delta V\left(\overline{\mathbf{u}}_{j}\right)\right)\right] .
\end{aligned}
$$

Here, $\Delta V\left(\overline{\mathbf{u}}_{j}\right)$ must be determined via 23.

$$
\Delta V\left(\overline{\mathbf{u}}_{j}\right)=\left.\frac{\hbar^{2}}{8 m} \sum_{\alpha, \beta, \gamma}\left(\frac{\partial}{\partial u_{\alpha}} \frac{\partial u_{\beta}}{\partial u_{\gamma}}\right)\left(\frac{\partial}{\partial u_{\beta}} \frac{\partial u_{\alpha}}{\partial u_{\gamma}}\right)\right|_{\mathbf{u}=\overline{\mathbf{u}}_{j}}=\frac{\hbar^{2}}{8 m\left(\overline{\mathbf{u}}_{j}\right)^{4}} .
$$

I perform a time-substitution on midpoints according to $s_{j}-s_{j-1}=\Delta s_{j}=\epsilon / 4\left(\overline{\mathbf{u}}_{j}\right)^{2}$ and obtain

$$
K_{l}^{C}\left(r^{\prime \prime}, r^{\prime} ; T\right)=\int_{\mathbb{R}} \frac{d E}{2 \pi \mathrm{i}} \mathrm{e}^{-\mathrm{i} E T / \hbar} G_{l}^{C}\left(r^{\prime \prime},{ }^{\prime} r ; E\right)
$$


with the energy-dependent Green functions $G_{l}^{C}\left(r^{\prime \prime},{ }^{\prime} r ; E\right)$ given by

$$
\begin{aligned}
& G_{l}^{C}\left(r^{\prime \prime},{ }^{\prime}, r ; E\right)=\frac{1}{\sqrt{r^{\prime} r^{\prime \prime}}} \int_{\mathbb{R}} d y^{\prime \prime} \frac{\mathrm{i}}{\hbar} \int_{0}^{\infty} d s^{\prime \prime} \mathrm{e}^{\mathrm{i} e^{2} s^{\prime \prime} / \hbar} \\
& \quad \times \int_{\mathbf{u}(0)=\mathbf{u}^{\prime}}^{\mathbf{u}\left(s^{\prime \prime}\right)=\mathbf{u}^{\prime \prime}} \mathcal{D} \mathbf{u}(s) \exp \left[\frac{\mathrm{i}}{\hbar} \int_{0}^{s^{\prime \prime}}\left(\frac{m}{2} \dot{\mathbf{u}}^{2}+E|\mathbf{u}|^{2}-\hbar^{2} \frac{(2 l+1)^{2}}{2 m|\mathbf{u}|^{2}}\right) d s\right] .
\end{aligned}
$$

The path integral (11) is the path integral of a radial harmonic oscillator in $\mathbb{R}^{2}$. We introduce polar coordinates

$$
\begin{aligned}
& u_{1}=v \cos \frac{\alpha}{2}, \\
& u_{2}=v \sin \frac{\alpha}{2},
\end{aligned} \quad 0 \leq \alpha<4 \pi, v=|\mathbf{u}|
$$

use the expansion of plane waves into circular waves in terms of modified Bessel functions $I_{\nu}$

$$
\mathrm{e}^{z \cos \psi}=\sum_{\nu \in \mathbb{Z}} \mathrm{e}^{\mathrm{i} \nu \psi} I_{\nu}(z)
$$

and the asymptotic expansion [10, 19, 20]

$$
I_{\nu}\left(\frac{z}{\epsilon}\right) \stackrel{(\epsilon \rightarrow 0)}{\simeq} \sqrt{\frac{\epsilon}{2 \pi z}} \exp \left(\frac{z}{\epsilon}-\frac{\nu^{2}-1 / 4}{2 z}\right)
$$

and obtain after an $\alpha$-angular integration over $4 \pi$ (we set $\omega=\sqrt{-E / 2 m}$ )

$$
\begin{aligned}
& G_{l}^{C}\left(r^{\prime \prime}, r^{\prime} ; E\right)=\frac{2}{\sqrt{r^{\prime} r^{\prime \prime}}} \int_{0}^{\infty} \frac{\mathrm{e}^{\mathrm{i} e^{2} s^{\prime \prime} / \hbar} s^{\prime \prime}}{\sqrt{v^{\prime} v^{\prime \prime}}} \\
& \quad \times \int_{v(0)=v^{\prime}}^{v\left(s^{\prime \prime}\right)=v^{\prime \prime}} \mathcal{D} v(s) \exp \left[\frac{\mathrm{i}}{\hbar} \int_{0}^{s^{\prime \prime}}\left(\frac{m}{2}\left(\dot{v}^{2}-\omega^{2} v^{2}\right)-\hbar^{2} \frac{(2 l+1)^{2}-1 / 4}{2 m v^{2}}\right) d s\right] \\
& =\int_{0}^{\infty} \frac{\mathrm{e}^{\mathrm{i} e^{2} s^{\prime \prime} / \hbar} s^{\prime \prime}}{\sqrt{v^{\prime} v^{\prime \prime}}} \frac{m \omega \sqrt{v^{\prime} v^{\prime \prime}}}{\mathrm{i} \hbar \sin \omega s^{\prime \prime}} \exp \left[\frac{\mathrm{i} m \omega}{2 \hbar}\left(v^{\prime 2}+v^{\prime \prime 2}\right) \cot \omega s^{\prime \prime}\right] I_{2 l+1}\left(\frac{m \omega v^{\prime} v^{\prime \prime}}{\mathrm{i} \hbar \sin \omega s^{\prime \prime}}\right) \\
& =\frac{1}{\hbar} \frac{1}{r^{\prime} r^{\prime \prime}} \sqrt{-\frac{m}{2 E}} \frac{\Gamma(1+l-\kappa)}{(2 l+1) !} W_{\kappa, l+\frac{1}{2}}\left(\sqrt{-8 m E} \frac{r_{>}}{\hbar}\right) M_{\kappa, l+\frac{1}{2}}\left(\sqrt{-8 m E} \frac{r_{<}}{\hbar}\right) .
\end{aligned}
$$

Here, use has been made of the well-known path integral identity for the radial harmonic oscillator [2],

$$
\begin{aligned}
& \int_{r\left(t^{\prime}\right)=r^{\prime}}^{r\left(t^{\prime \prime}\right)=r^{\prime \prime}} \mathcal{D} r(t) \exp \left\{\frac{\mathrm{i}}{\hbar} \int_{t^{\prime}}^{t^{\prime \prime}}\left[\frac{m}{2}\left(\dot{r}^{2}-\omega^{2} r^{2}\right)-\hbar^{2} \frac{\lambda^{2}-1 / 4}{2 m r^{2}}\right] d t\right\} \\
& =\sqrt{r^{\prime} r^{\prime \prime}} \frac{m \omega}{\mathrm{i} \hbar \sin \omega T} \exp \left[-\frac{m \omega}{2 \mathrm{i} \hbar}\left(r^{\prime 2}+r^{\prime \prime 2}\right) \cot \omega T\right] I_{\lambda}\left(\frac{m \omega r^{\prime} r^{\prime \prime}}{\mathrm{i} \hbar \sin \omega T}\right),
\end{aligned}
$$


the integral representation 24

$$
\begin{aligned}
M_{\kappa \mu}(a) W_{\kappa \mu}(b) & =\frac{\sqrt{a b}}{\left.\Gamma) \mu-\kappa+\frac{1}{2}\right)} \int_{0}^{\infty} d s\left(\operatorname{coth} \frac{s}{2}\right)^{2 \kappa} \\
& \times \exp \left(-\frac{1}{2}(a+b) \cosh s\right) I_{2 \mu}(\sqrt{a b} \sinh s)
\end{aligned}
$$

and I have used the usual abbreviation $\kappa=\left(e^{2} / \hbar\right) \sqrt{-m / 2 E} . \quad r_{\gtrless}$ denotes the greater/lesser of $r^{\prime}, r^{\prime \prime}$. From the poles of the Green function $G_{l}^{C}(E)$ we immediately derive the Coulomb bound state spectrum

$$
E_{n}^{C}=-\frac{m e^{4}}{2 \hbar^{2}(n+l+1)^{2}}, \quad n \in \mathbb{N}_{0} .
$$

The bound state wave-functions are obtained in the usual way by considering the residua in (15), and the scattering states are obtained by an analysis of the cuts of $G_{l}^{C}(E)$.

I have therefore obtained the radial Coulomb Green function by an alternative method by using a singular version of the Levi-Civita transformation. The usual Levi-Civita transformation [7, 19] can be used in the evaluation of the twodimensional Coulomb path integral. Actually, the Levi-Civita transformation maps $\mathbb{R}^{2} \mapsto \mathbb{R}^{2}: x=\xi^{2}-\eta^{2}, y=2 \xi \eta$, and the coordinates $\xi, \eta$ correspond to parabolic coordinates in $\mathbb{R}^{2}$ in which the Coulomb problem is also separable, e.g. [15] for an extensive discussion. For the singular Kustaanheimo-Stiefel transformation in the case of the three-dimensional Coulomb potential, the resemblance to parabolic coordinates can also be recognised, and in the present case as well; however, in both cases we must integrate over an additional variable. In all cases we encounter a path integral for a (radial) harmonic oscillator. Whereas in the three-dimensional Coulomb potential the non-uniqueness of the transformation appears quite natu-

rally and leads to an isotropic harmonic oscillator in $\mathbb{R}^{4}$, the transformation (7) looks somewhat circumstantial, because the one-dimensional transformation $r=u^{2}$ $(u>0)$ also does the job in a much simpler way [25]. The virtue of (7) lies in the fact that it demonstrates once again the importance of the Kustaanheimo-Stiefel transformation, it points out that singular transformations also work in the path integral, and that it emphasizes a singular transformation may be in other cases the only means which work.

\section{Acknowledegement}

I would like to thank the members of the Joint Institute for Nuclear Research, Dubna, in particular G.Pogosyan, for the warm hospitality during my stay in Dubna. I also thank F.Steiner for useful discussions on the Kepler-Coulomb problem. Financial support from the Heisenberg-Landau program is greatfully acknowledged. 


\section{References}

[1] Feynman, R.P.: Space-Time Approach to Non-Relativistic Quantum Mechanics. Rev.Mod.Phys. 20 (1948) 367-387.

[2] Peak, D., Inomata, A.: Summation Over Feynman Histories in Polar Coordinates. J.Math.Phys. 10 (1969) 1422-1428.

[3] Böhm, M., Junker, G.: Path Integration Over Compact and Noncompact Rotation Groups. J.Math.Phys. 28 (1987) 1978-1994.

[4] Grosche, C., Steiner, F.: How to Solve Path Integrals in Quantum Mechanics. J.Math.Phys. 36 (1995) 2354-2385.

[5] Grosche, C., Steiner, F.: Handbook of Feynman Path Integrals. Springer, Berlin, Heidelberg, 1998.

[6] Duru, I.H., Kleinert, H.: Solution of the Path Integral for the H-Atom. Phys.Lett. B 84 (1979) 185-188.

[7] Duru, I.H., Kleinert, H.: Quantum Mechanics of H-Atoms From Path Integrals. Fortschr.Phys. 30 (1982) 401-435.

[8] Kustaanheimo, P., Stiefel, E.: Perturbation Theory of Kepler Motion Based on Spinor Regularization. J.Rein.Angew.Math. 218 (1965) 204-219.

[9] Castrigiano, D.P.L., Stärk, F.: New Aspects of the Path Integrational Treatment of the Coulomb Potential. J.Math.Phys. 30 (1989) 2785-2788.

[10] Fischer, W., Leschke, H., Müller, P.: Changing Dimension and Time: Two Well-Founded and Practical Techniques for Path Integration in Quantum Physics. J.Phys.A: Math.Gen. 25 (1992) 3835-3853.

[11] Grosche, C.: Coulomb Potentials by Path-Integration. Fortschr.Phys. 40 (1992) 695737.

[12] Grosche, C.: Path Integral Solution of a Non-Isotropic Coulomb-Like Potential. Phys.Lett. A 165 (1992) 185-190.

[13] Grosche, C.: Path Integral Solution of Two Potentials Related to the $\mathrm{SO}(2,1)$ Dynamical Algebra. J.Phys.A: Math.Gen. 26 (1993) L279-L287.

[14] Grosche, C.: Path Integrals, Hyperbolic Spaces, and Selberg Trace Formula. World Scientific, Singapore, 1996.

[15] Grosche, C., Pogosyan, G.S., Sissakian, A.N.: Path Integral Discussion for Smorodinsky-Winternitz Potentials: I. Two- and Three-Dimensional Euclidean Space. Fortschr.Phys. 43 (1995) 453-521.

[16] Grosche, C., Steiner, F.: Path Integrals on Curved Manifolds. Zeitschr.Phys. C 36 (1987) 699-714.

[17] Ho, R., Inomata, A.: Exact Path Integral Treatment of the Hydrogen Atom. Phys.Rev.Lett. 48 (1982) 231-234. 
[18] Hurwitz, A.: Mathematische Werke. Birkhäuser, Basel, 1933, II, pp.641.

[19] Inomata, A.: Exact Path-Integration for the Two Dimensional Coulomb Problem. Phys.Lett. A 87 (1982) 387-390.

[20] Inomata, A.: Alternative Exact-Path-Integral-Treatment of the Hydrogen Atom. Phys.Lett. A 101 (1984) 253-257.

[21] Kleinert, H.: Path Integrals in Quantum Mechanics, Statistics and Polymer Physics. World Scientific, Singapore, 1990.

[22] Young, A., DeWitt-Morette, C.: Time Substitution in Stochastic Processes as a Tool in Path Integration. Ann.Phys. 169 (1986) 140-116.

[23] Lee, T.D.: Particle Physics and Introduction to Field Theory. Harwood Academic Publishers, Chur, 1981.

[24] Gradshteyn, I.S., Ryzhik, I.M.: Table of Integrals, Series, and Products. Academic Press, New York, 1980.

[25] Inomata, A., Kayed, M.A.: Exact Path-Integral Solution of the Dirac-Coulomb Problem. Phys. Rev.Lett. 53 (1984) 107-110. 